\title{
Heat Transfer Augmentation Using Dissimilar Porous Baffles in a Backward-Facing Step Flow
}

\author{
James Arthur ${ }^{1}$ \\ ${ }^{1}$ Bucknell University \\ Mechanical Engineering Department \\ 1 Dent Drive, Lewisburg, PA, United States of America \\ james.arthur@bucknell.edu
}

\begin{abstract}
This paper reports a numerical investigation of the use of a pair of baffles of dissimilar porosity as heat augmentation devices in a backward-facing step flow in a two-dimensional channel. The specific goal of this study is to explore the possibility of providing a pressure penalty-free heat transfer enhancement using such a pair of porous baffles in a laminar forced convection flow. The numerical simulations are done using a finite element-based COMSOL Multiphysics commercial software. The parameters studied include Reynolds number $(100,200,300,400,500)$, porous baffle solid volume fraction $\phi(0.031,0.125,0.500)$, and the porous baffle streamwise location $(0.5,1.0,1.5,2.0,2.5,3.0$ times the channel height). The results show that the porous baffles result in increments of the local peak Nusselt Numbers and global Nusselt numbers by up to $200 \%$ and $85 \%$ respectively with an increase in Reynolds number from 100 to 500. For the same pressure drop requirement, the average convection to conduction heat transfer is up to $16 \%$ better when a pair of baffles are used, compared to an unobstructed flow. For the channel tested in this work, the best location for optimal heat augmentation with least pressure penalty is reached both porous baffles are located at a streamwise distance $\sim 2$ times the channel's expanded height.
\end{abstract}

Keywords: porous baffles $\cdot$ heat transfer augmentation $\cdot$ backward-facing step $\cdot$ finite element analysis

\section{Introduction}

There are several engineering systems that are characterized by abrupt expansions. Compact electronic devices, for example, may be designed with step-like expansions for the convenient installation of electronic components. To avoid overheating of such electronic systems, such systems would require an optimal design that assures heat transfer through forced convection. Solid flow-obstructing and flow-modification devices called baffles have been proposed for such purpose. However, they have been known to incur significant pressure penalty for flows through them. Consequently, in a bid to cut back on the differential pressure costs, alternative porous designs of such baffles have been suggested.

The inspiration for the use of porous baffles in sudden expansion (or backward-facing step) channel flows stems from the performance of porous blocks in ordinary channel flows, and porous floor segments in backward-facing step flows. Huang and Vafai [1] studied the effects of the use of intermittently placed porous blocks in a two-dimensional channel. In a later study, Huang et al. [2] carried out a numerical analysis on the use of multiple heated blocks in a channel using porous covers. They showed that the recirculation caused by porous-covering blocks significantly enhance the heat transfer rates. Martin et al. [3] and Abu-Hijleh [4] also looked into the use of porous floor segments for heat transfer augmentation in a laminar backward-facing step flow. From their numerical analysis, Martin et al. [3] concluded that the porous inserts could enhance heat transfer by reducing or eliminating the recirculation zone on the lower wall of the backward-facing step flow. Abu-Hijleh [4] noted that by using the floor segments, the maximum local convection-to-conduction heat transfer ratio increased by as much as $170 \%$.

Despite the prospective value of porous baffles, the body of literature supporting its study and utilization as a heat enhancement device in backward-facing step flows has been rather limited. In one of relatively few studies, Cheng and Tsay [5] compared the effects of the use of solid and slotted baffles in laminar forced convection flow over a backward-facing step. They observed that for the solid baffle, the heat transfer improvement peaks at $230 \%$, while that of the slotted ones is at $190 \%$. However, the penalty of increase in pressure drop is much higher in the case of solid baffles. Li et al. [6] also modelled such a backward-facing step flow to study the heat and laminar flow field. A porous baffle was placed on the wall opposing that of the step. That work ultimately showed that compared to a flow without baffles, the inclusion of porous 
baffles resulted in about 35\% improvement of heat transfer at a Reynolds number of 500. Very recently, Zhao [7] studied the fluid flow and heat transfer over a backward-facing step with a porous insert mounted just after the step. That work showed that even a porous baffle with low permeability could enhance heat transfer, while incurring only a little higher pressure drop. While Zhao's study appears to be the only one that suggests no penalty using a baffle, the Reynolds number coverage in that study limited. This calls for the need to design better baffles of a wider range of utility.

It is in this light that this work investigates the use of porous baffles as heat augmentation devices in a backwardfacing step flow in a two-dimensional channel. To the author's knowledge, it is the first study that considers the use of a pair of adiabatic baffles of dissimilar porosity to achieve heat transfer augmentation. The uniqueness of this study also lies in its goal to provide a pressure penalty-free heat transfer enhancement in a laminar forced convection flow. This numerical study is accomplished using a finite element-based COMSOL Multiphysics commercial software. The parameters studied include Reynolds number, porous baffle permeability, and porous baffle location. The thermal-fluid characteristics are analysed using streamlines, contours, Nusselt numbers, and a performance number (the ratio of the Nusselt number improvement to pressure drop increment relative to an empty backward-facing step flow case).

\section{Numerical Model and Method}

The system under study is shown in Fig. 1 along with the Cartesian coordinate system used in this study. As shown in Fig. 1, the origin of the streamwise axis $x=0$ is positioned at the backward-facing step of the channel. The origin of the transverse direction $y=0$ is also located at the bottom wall of the upstream channel. In the following subsections, the geometric model, physical laws, solution procedure and the validation methods used are described.

\subsection{Physical Problem}

The test system consists of a planar channel with a pair of porous baffles mounted close to the upper and lower walls of the test channel. The channel has an upstream height $h(=0.02 \mathrm{~m})$ expanding by a backward-facing step of height $S(=0.02 \mathrm{~m})$ to a downstream height $H(=0.04 \mathrm{~m})$. The upstream length $L_{u}$ is fixed at $2.5 H$ while the expanded channel downstream is of length $L_{d}=7.5 \mathrm{H}$. The porous baffles are modelled as square arrays of two-dimensional circular rods of diameter $d=0.025 \mathrm{H}$. By varying the distance between rod centres $l$, various solid volume fractions $\phi=$ $\pi d^{2} /\left(4 l^{2}\right)=0.031,0.125$, and 0.500 of the porous baffles are accomplished. For each test, the porous baffle model mounted close to the upper channel wall (called the upper porous baffle), and that mounted close to the lower channel wall (called lower porous baffle) are of different $\phi$. However, the baffles are designed to be of the same length $L_{p}=$ $0.276 \mathrm{H}$ and height $H_{p}=0.150 \mathrm{H}$. The baffles are located such that the centre of the most upstream rod is at a variable streamwise location $x / H(=0.5,1.0,1.5,2.0,2.5,3.0)$ behind the step. A gap distance of $d$ is maintained between the channel wall and the centre of the most immediate row of rods of each porous baffle.

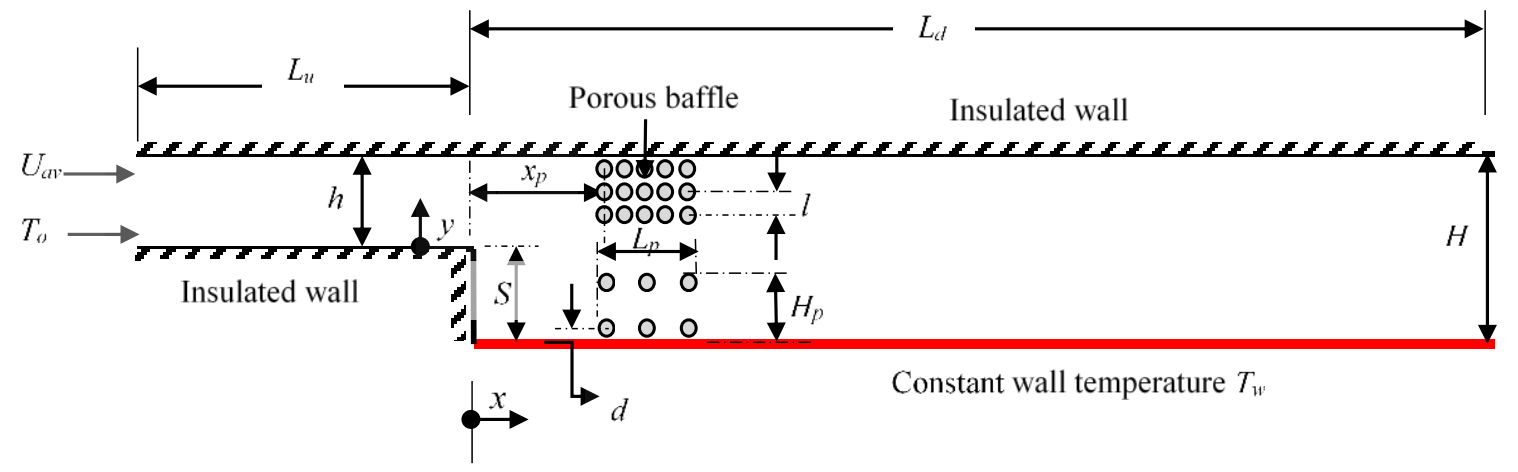

Fig. 1: Schema of the computational model.

\subsection{Physics of Model}

The assumptions underlying the model are that of a planar steady-state incompressible Newtonian flow, without body forces. The porous media are taken to be isotropic, adiabatic, rigid and fully saturated with the fluid in the test 
channel. The fluid and thermal flow fields are obtained through the numerical solution of the steady laminar governing equations in the streamwise $(x)$ and transverse $(y)$ directions. The governing equations are the following:

Continuity equation -

$$
\frac{\partial}{\partial x}(\rho u)+\frac{\partial}{\partial y}(\rho v)=0
$$

Navier-Stokes equation in the $x$ and $y$ directions, respectively -

$$
\begin{aligned}
\frac{\partial}{\partial x}(\rho u u)+\frac{\partial}{\partial y}(\rho u v) & =-\frac{\partial p}{\partial x}+\mu\left(\frac{\partial^{2} u}{\partial x^{2}}+\frac{\partial^{2} u}{\partial y^{2}}\right) \\
\frac{\partial}{\partial x}(\rho u v)+\frac{\partial}{\partial y}(\rho v v) & =-\frac{\partial p}{\partial y}+\mu\left(\frac{\partial^{2} v}{\partial x^{2}}+\frac{\partial^{2} v}{\partial y^{2}}\right)
\end{aligned}
$$

Energy equation -

$$
\frac{\partial}{\partial x}\left(\rho C_{p} u T\right)+\frac{\partial}{\partial y}\left(\rho C_{p} v T\right)=k\left(\frac{\partial^{2} T}{\partial x^{2}}+\frac{\partial^{2} T}{\partial y^{2}}\right)
$$

In Eqs. (1) to (4), $u$ and $v$ denote velocity components in the $x$ and $y$ coordinate directions, and $\mu, \rho, p, T, k, C_{p}$ are respectively dynamic viscosity, density, pressure, temperature, thermal conductivity and specific heat capacity. The working fluid is specified as air with properties varying with temperature, as stipulated in reference [8]. The porous baffles are assigned material properties of Aluminium as given in reference [8].

For each run of the numerical simulations, fluid flows into the channel at a normal inflow velocity $U_{a v}$ and constant inlet temperature $T_{o}=298 \mathrm{~K}$. The exit boundary is defined by a constant pressure value. Additionally, no-slip conditions are assigned at solid walls (velocities set at zero), a constant temperature, $T_{w}=323.15 \mathrm{~K}$ is applied at the lower wall of the expanded channel, and the porous baffle rods as well as all other walls are set at thermally adiabatic conditions.

\subsection{Numerical Solution, Mesh Independence Study and Validation}

The model was discretized into unstructured triangular and quadrilateral elements, taking into consideration the relevant physics under study. It should be noted then that at the porous medium and wall domains, very fine meshes were applied. Solutions of the conjugate fluid flow and heat transfer domains were obtained using a two-dimensional laminar segregated solver. For all the test cases, the residuals of the governing equations were set at a tolerance of $10^{-8}$.

An extensive mesh independence study was carried out to assess the sensitivity of the numerical solutions to variations of mesh elements and sizes. Summary results of three levels of mesh refinement are shown in Table 1 for a backward-facing flow with or without a pair of baffles installed close to the lower and upper walls. The tests were carried out over a wide range of Reynolds number $\left(R e=\rho U_{a v} H / \mu=100,200,300,400,500\right.$; where dynamic viscosity $\mu=1.849 \times 10^{-5} \mathrm{~Pa} \mathrm{~s}$ and density $\rho=1.184 \mathrm{~kg} / \mathrm{m}^{3}$ ). The results were evaluated by comparing values of the average Nusselt number $\overline{N u}$. The average Nusselt number is a surface-averaged parameter defined as

$$
\overline{N u}=\int_{0}^{L_{d}} N u(x) d x
$$

In Eq. (5), $N u$ is the local Nusselt number computed with respect to the lower wall, and it is also defined as 


$$
N u=\frac{-\left.H \frac{\partial T(x, y)}{\partial y}\right|_{y=-S}}{T_{w}-T_{o}}
$$

As the relative deviations between $\mathrm{M} 2$ and $\mathrm{M} 3$ are less than $1 \%$, those results are indeed independent of mesh sizes. Thus, it was concluded that a minimum elemental size of $0.00002 \mathrm{H}$, and a maximum elemental size of $0.025 \mathrm{H}$ was sufficiently fine to resolve the physics of the flow. This resulted in total number of elements ranging from 111,414 to 153,285 , depending on the complexity of the computational domain.

Table 1: Summary of mesh independence study for backward-facing step flow with or without a pair of porous baffles. This was done using assessments of the averaged Nusselt number.

\begin{tabular}{|c|c|c|c|c|c|c|c|}
\hline $\begin{array}{c}\text { Mesh } \\
\text { Refinement }\end{array}$ & $N_{\text {elements }}$ & $\begin{array}{l}\text { Minimum Element } \\
\text { Spacing (m) }\end{array}$ & $R e=100$ & $R e=200$ & $R e=300$ & $R e=400$ & $R e=500$ \\
\hline \multicolumn{8}{|c|}{ With Porous Baffles } \\
\hline & & & \multicolumn{5}{|c|}{$\overline{N u}$} \\
\hline M1 & 52372 & $1.60 \mathrm{E}-05$ & 2.0317 & 2.6693 & 3.1223 & 3.4813 & 3.7954 \\
\hline M2 & 80081 & $6.00 \mathrm{E}-06$ & 2.0334 & 2.6704 & 3.1206 & 3.4758 & 3.7801 \\
\hline M3 & 153746 & 8.00E-07 & 2.0341 & 2.6710 & 3.1205 & 3.4748 & 3.7780 \\
\hline \multicolumn{8}{|c|}{ Without Porous Baffles (Unobstucted) } \\
\hline & & & \multicolumn{5}{|c|}{$\overline{N u}$} \\
\hline M1 & 19729 & $1.60 \mathrm{E}-05$ & 1.9520 & 2.4134 & 2.6640 & 2.8457 & 3.0212 \\
\hline M2 & 46791 & $6.00 \mathrm{E}-06$ & 1.9529 & 2.4109 & 2.6522 & 2.8163 & 2.9695 \\
\hline M3 & 111414 & $8.00 \mathrm{E}-07$ & 1.9534 & 2.4107 & 2.6502 & 2.8107 & 2.9541 \\
\hline
\end{tabular}

The numerical procedure was validated using the laminar forced convection backward-facing step flow numerical study by Kumar and Dhiman [9]. The case of a two-dimensional channel flow with an adiabatic circular cylinder installed at $x / S=0.6$ and $y / S=0.6$ was tested. In Table 2 , summary results of the maximum Nusselt number $N u_{\max }$ computations are presented. The results show that the data obtained from the current procedure and that of Kumar and Dhiman [9] are within $2 \%$ in relative deviations.

Table 2: Results of maximum local Nusselt number $N u_{\max }$ computations of the current method and that of with results of simulations by Kumar and Dhiman [9] are compared.

\begin{tabular}{|c|c|c|}
\hline Reynolds Number & \multicolumn{2}{|c|}{$N u_{\max }$} \\
\hline & Present Study & Kuma and Dhiman [9] \\
\hline 100 & 3.4432 & $3.5613 \pm 1.9927 \%$ \\
\hline 150 & 4.4485 & $4.5994 \pm 1.9927 \%$ \\
\hline 200 & 5.5282 & $5.4203 \pm 1.9927 \%$ \\
\hline
\end{tabular}

\subsection{Substantive Tests}

In order to test the utility of using a pair of dissimilar porous baffles, a number of parameters is varied. A primary parameter of study is the Reynolds number of the flow $\left(\operatorname{Re}=\rho U_{a v} H / \mu=100,200,300,400,500\right)$. The Reynolds number is computed using air property values evaluated at the inlet temperature $T_{o}$ of $298 \mathrm{~K}$ (i.e. dynamic viscosity $\mu$ $=1.849 \times 10^{-5} \mathrm{~Pa} \mathrm{~s}$ and density $\left.\rho=1.184 \mathrm{~kg} / \mathrm{m}^{3}[10]\right)$. The other parameters tested are those associated with the porous baffles. Thus, permutations of baffle solid volume fraction $\phi(0.031,0.125,0.500)$ of the lower and upper porous baffles, porous baffle location $x / H(=0.5,1.0,1.5,2.0,2.5,3.0)$ are explored. Two base conditions consisting of (1) a 
backward-facing step flow without a baffle, and (2) the case of a pair of baffles of solid volume fraction 0.031, are also computed. The numerical results of the tests are then assessed using streamlines, temperature contours, local and average Nusselt number computations. Other parameters used include a Nusselt number ratio defined as

$$
\overline{N u}^{*}=\frac{\overline{N u}}{\overline{N u}_{b}}
$$

where $\overline{N u} \bar{u}_{b}$ is the average Nusselt number for a corresponding base backward-facing step flow in which there are no porous baffles. Additionally, a pressure drop ratio and the performance number is employed. The pressure drop ratio is also defined as

$$
\Delta p^{*}=\frac{\Delta p}{\Delta p_{b}}
$$

where $\Delta p$ and $\Delta p_{b}$ are respectively the pressure drops between $x / H=-2.5$ and $x / H=7.5$ with and without porous baffles. The performance number $P N$ is defined as

$$
P N=\frac{\overline{N u}^{*}}{\Delta p^{*}}
$$

\section{Results and Summary Discussion}

The general effect of introducing a pair of dissimilar porous media in the backward facing flow is clear from the streamlines presented in Fig. 2. Depending on the Reynolds number and the solid volume fraction of the baffle at the upper wall, a secondary recirculation zone appears, and subsequently grows close to the upper wall. The size of the recirculation zone just behind the step is also markedly reduced, depending on the location of the porous baffle on the lower wall. The result of all these are significant changes in heat transfer and pressure drop characteristics such as those briefly summarized in the subsections that follow.

\subsection{Reynolds Number}

The effects of Reynolds number on the backward-facing step flows are shown in Figs. 2 and 3. In the streamlines of the former, the effect of the upper wall baffle appears to be minimal at Reynolds number $R e=100$. This is because the solid volume fraction of the baffle is sufficiently low, and the inertia of the flow is relatively low. As indicated in the normalized temperature contour plots of Fig. 2, at that low speed, the temperature gradient before and behind the lower porous baffle is relatively thick, implying that the heat transfer is reduced. However, upon increasing the Reynolds number, the secondary recirculation zone close to the upper wall appears, and subsequently grows with the recirculation zones behind the step. Due to the presence of the lower porous baffle, this recirculation zonal growth rather extends in height. The result of that growth is an enhanced mixing and a decrease in the temperature gradients, resulting in an augmented heat transfer. Thus, as shown in Fig. 3, the local peak Nusselt Numbers and global Nusselt numbers increase monotonically by up to $200 \%$ and $85 \%$ respectively.

Increasing the Reynolds number from 100 to 500 however incurs an increased pressure differential (and pump power) requirement. The increase in pressure drop at $R e=500$ can be as high as six times the value at $R e=100$. While high, this value is lower than the value reported by $\mathrm{Li}$ et al. [6] who studied a similar Reynolds number range for a single porous baffle mount. The increase in pressure drop for the case of a dissimilar pair of porous baffle installations is however at a lower rate than that of an unobstructed flow. Thus, as shown in the performance number $(P N)$ plots in Fig. 3(d), for the same pressure drop requirement, the average convection to conduction heat transfer is up to $16 \%$ better when a pair of baffles are used. 
(a) $\operatorname{Re}=100$

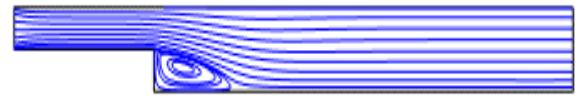

(d) $R e=100$

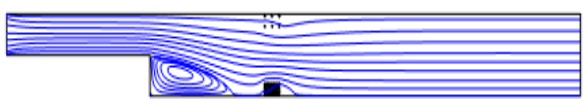

(g) $R e=100$ (b) $R e=300$

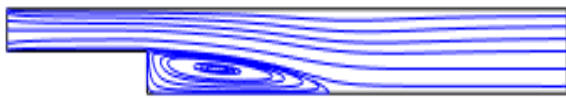

(e) $R e=300$

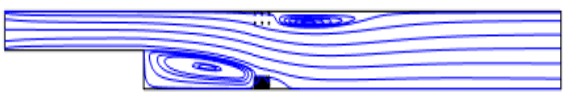

(h) $R e=300$

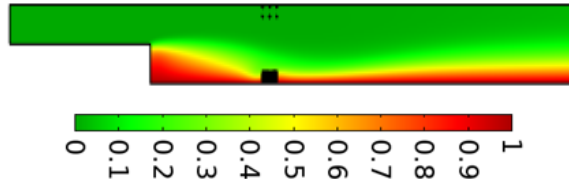

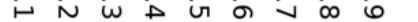

(c) $R e=500$

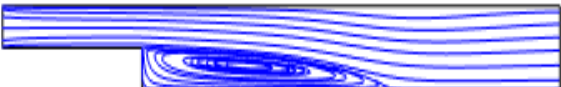

(f) $R e=500$

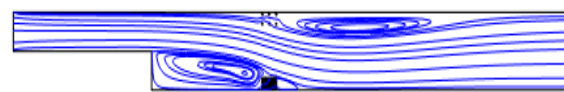

(i) $\operatorname{Re}=500$

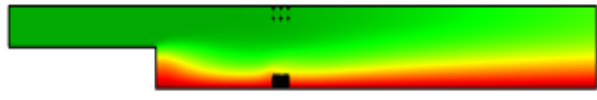

Fig. 2: Effect of porous baffles and Reynolds number shown using streamlines in (a $-\mathrm{f})$ and normalized temperature contours [ $(T$ $\left.\left.T_{o}\right) /\left(T_{w}-T_{o}\right)\right]$ in $(\mathrm{g}-\mathrm{i})$. Plots $(\mathrm{a}-\mathrm{c})$ are for unobstructed case; $(\mathrm{d}-\mathrm{i})$ are cases for which upper and lower porous baffles are of solid volume fractions $0.031,0.50$ respectively.
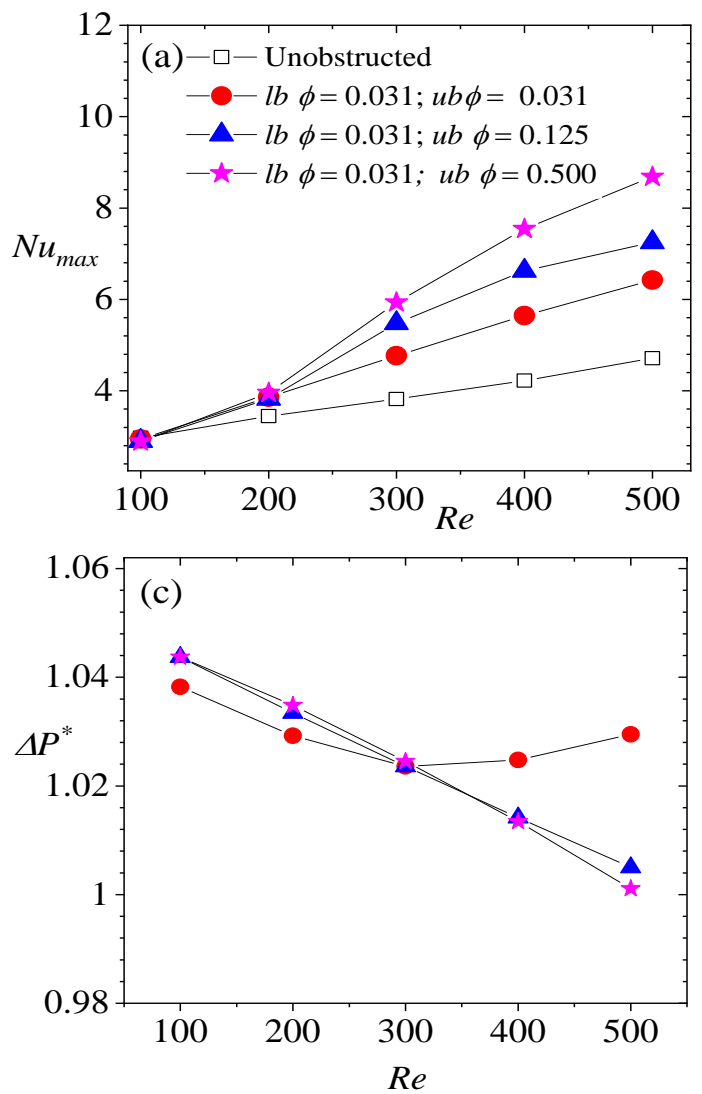
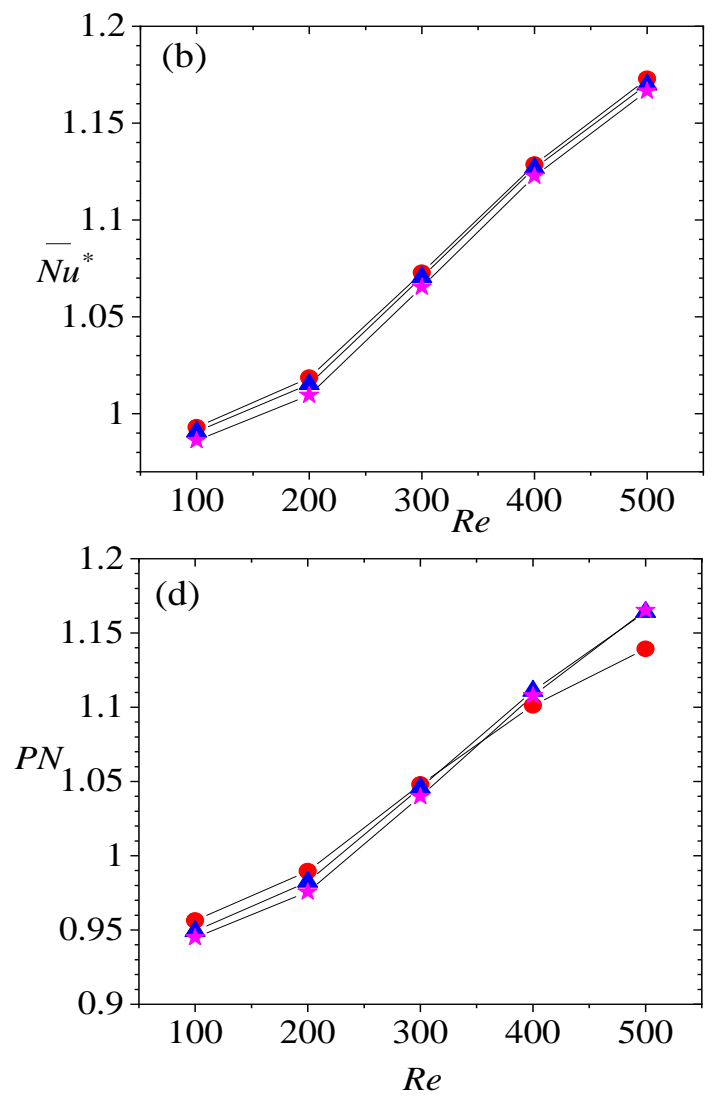

Fig. 3: Effect of Reynolds number demonstrated using (a) local peak Nusselt number, (b) Nusselt number ratio, (c) pressure drop ratio and (d) performance number. Note that $l b$ and $u b$ in legend are respectively baffles on lower and upper channel walls. 


\subsection{Solid Volume Fraction of Baffle}

At Reynolds number $R e>200$, the installation of the baffles guarantees an effective improvement in convection, regardless of the value of the solid volume fraction $\phi$. As shown in Fig. 3, this increment is up to 84\% for the local peak Nusselt numbers, and up to $\sim 20 \%$ in the average Nusselt numbers. It should however be noted that while the pressure differentials are greater in the flows with baffles (as opposed to the unobstructed flow), the deviation between the two drops monotonically with Reynolds number, especially if the baffles are dissimilar in $\phi$ (Fig. 3(c)). Consequently, the performance numbers also improve with the use of porous baffles.

It is further noted however in Fig. 4 that while the modification of $\phi$ in the lower porous baffle result in more changes in local Nusselt number than the modification of $\phi$ the upper porous baffle, the effects of either modifications are relatively insignificant in terms of the average Nusselt number. Thus, $\phi$ appears to have relatively insignificant bearing on the performance numbers of the flow arrangements with baffles.
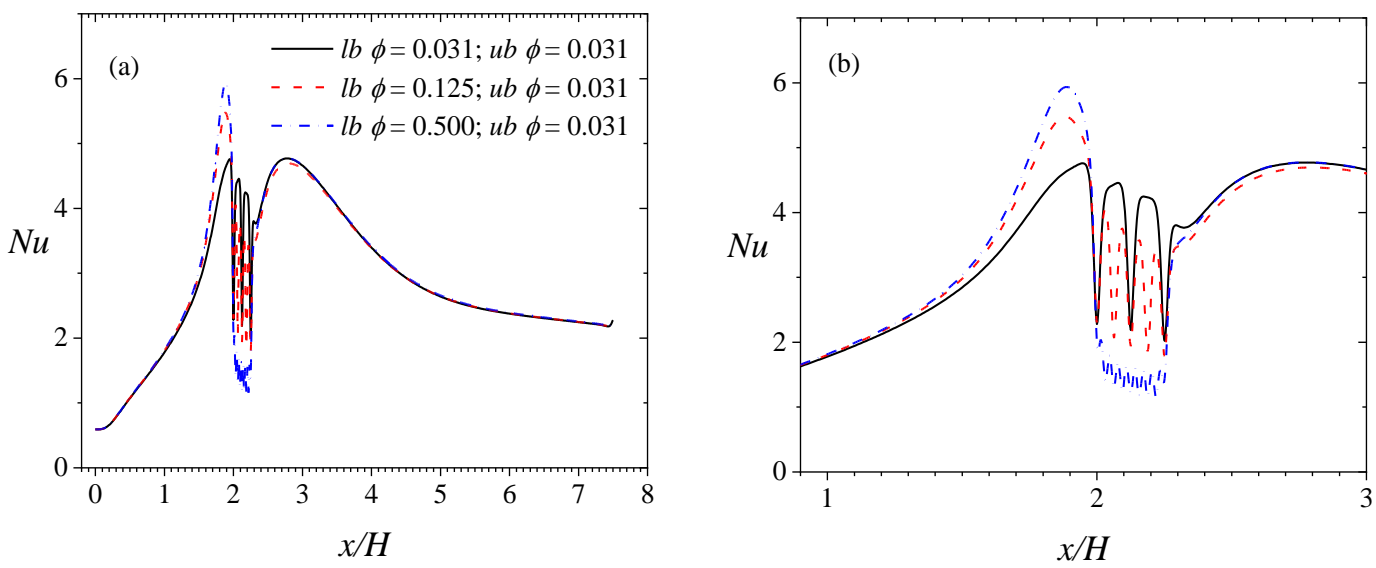

Fig. 4: Effect of dissimilarity of porous baffles tested using local peak Nusselt number distributions for various permutations of the upper baffle for (a) the entire length of the expanded channel, and (b) the upstream portion of the channel where the porous baffle is located for the same test conditions of (a). Note that $l b$ and $u b$ in legend are respectively baffles on lower and upper channel walls.
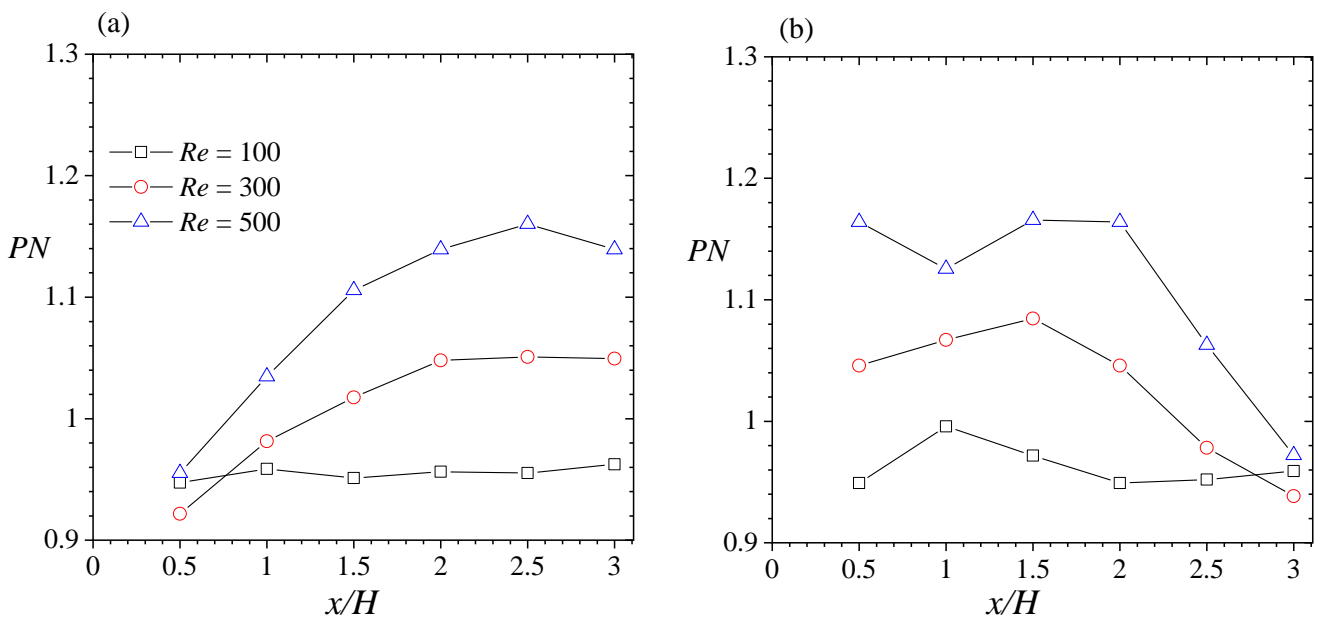

Fig. 5: Effectiveness of baffle location measured by a performance number parameter $(P N)$ for: (a) the case of lower porous baffle moved from $x / H=0.5$ to 3 while the upper porous baffle is kept at $x / H=2$. (b) the upper porous baffle moved from $x / H=0.5$ to 3 while the lower porous baffle is maintained at $x / H=2$. Note that for each plot, the upper and lower porous baffles are of solid volume fractions 0.031 and 0.125 respectively. 


\subsection{Streamwise location of Porous Baffle}

The modification in flow due to the streamwise location of porous baffles is most significantly discerned when the Reynolds number is greater than 200. As indicated in Fig. 5, the concomitant effects are that at such speeds, there is a of streamwise locations of porous baffles for which it is more beneficial to use a pair of porous baffles than an flow. This is particularly the case if on the one hand, the lower baffle is located at $1.5<x / H<3$ while the upper porous is set at $x / H=2$, or on the other hand, the upper baffle is located at $1<x / H<2$ while the lower porous baffle is fixed at $x / H=2$. Overall, it is apparent that the best streamwise location of both baffles for optimal heat enhancement with least pressure penalty is at $x / H \approx 2$.

\section{Conclusion}

In this work, the utility of a pair of dissimilar porous baffles in backward-facing step flows is explored using finite element analysis. This is done to provide a pressure penalty-free heat transfer augmentation in a laminar forced convection flow. The parameters studied include Reynolds number, porous baffle permeability, and porous baffle location. The results show that the porous baffles modify the thermal-fluid patterns of the flow in such a way that the recirculation region behind the step is reduced. Furthermore, a secondary recirculation region is formed behind the upper porous baffle that increases with Reynolds number. Consequently, the local peak Nusselt Numbers and global Nusselt numbers are increased by up to $200 \%$ and $85 \%$ respectively. Increasing the Reynolds number from 100 to 500 also tends to increase the pressure drop, albeit at a lower rate than that of an unobstructed flow. The result is a performance number up to $16 \%$, meaning that for the same pressure drop requirement, the average convection to conduction heat transfer is up to $16 \%$ better when a pair of baffles are used than the case of an unbaffled flow. This performance number is a direct function of the distance of the lower porous baffle from the backward-facing step. For the channel tested, the sweet spot for optimal heat augmentation with least pressure penalty is reached when both porous baffles are located at $x / H \approx 2$.

\section{Acknowledgement}

The financial support of this work through the C Graydon and Mary E Rogers Fellowship, is gratefully acknowledged.

\section{References}

[1] P.C. Huang, P. C. and K. Vafai, "Analysis of forced convection enhancement in a channel using porous blocks," Journal of Thermophysics and Heat Transfer, vol. 8, no. 3, pp. 563-573, 1994.

[2] P.C. Huang, C.F. Yang, J.J. Hwang, M.T. Chiu, "Enhancement of forced-convection cooling of multiple heated blocks in a channel using porous covers," International Journal of Heat and Mass Transfer, vol. 48, Issues 3-4, pp. 647 - 664, 2005.

[3] B. Abu-Hijleh, "Convection heat transferfrom a laminar flow over a 2-d backward facing step with asymmetric and orthotropic porous floor segments," Numerical Heat Transfer, Part A Applications, vol. 31, no. 3, pp. 325-335, 1997.

[4] A. R. Martin, C. Saltiel and W. Shyy, "Heat transfer enhancement with porous inserts in recirculating flows," J. Heat Transfer, vol. 120, no. 2, pp. 458-467, 1998.

[5] J.-C. Cheng and Y.-L. Tsay, "Effects of solid and slotted baffles on the convection characteristics of backward-facing step flow in a channel," Heat and Mass Transfer, vol. 42, pp. 843-852, 2006.

[6] C. Li, G. Cui, J. Zhai, S. Chen and Z. Hu, "Enhanced heat transfer and flow analysis in a backward-facing step using a porous baffle," Journal of Thermal Analysis and Calorimetry volume, vol. 141, pp. 1919-1932, 2020.

[7] Z. Zhao, "Numerical modeling and simulation of heat transfer and fluid flow in a two-dimensional sudden expansion model using porous insert behind that," Journal of Thermal Analysis and Calorimetry, vol. 141, pp. 1933-1942, 2020.

[8] COMSOL Multiphysics® v. 5.4. www.comsol.com. COMSOL AB, Stockholm, Sweden.

[9] A. Kumar and A. K. Dhiman, "Effect of a circular cylinder on separated forced convection at a backward-facing step," International Journal of Thermal Sciences, vol. 52, pp. 176-185, 2012. 